\section{(2) OPEN ACCESS}

Review

\title{
Prevalence of dementia in ischaemic or mixed stroke populations: systematic review and meta-analysis
}

\author{
Louise Craig ำ, ' Zhi Liang Hoo, ${ }^{1}$ Toh Zeng Yan, ${ }^{2}$ Joanna Wardlaw 두, \\ Terence J Quinn (1) ${ }^{1}$
}

- Additional supplemental material is published online only. To view, please visit the journal online (http://dx. doi.org/10.1136/jnnp-2020 325796).

${ }^{1}$ Institute of Cardiovascular and Medical Sciences, University of Glasgow College of Medical, Veterinary and Life Sciences, Glasgow, UK

${ }^{2}$ Geriatric Medicine, Hospital Kuala Lumpur, Kuala Lumpur, Malaysia

${ }^{3}$ Centre for Clinical Brain Sciences, University of Edinburgh, Edinburgh, UK

\section{Correspondence to}

Dr Terence J Quinn, Institute of cardiovascular and Medical Sciences, University of Glasgow College of Medical Veterinary and Life Sciences, Glasgow, Glasgow, UK; Terry.quinn@ glasgow.ac.uk

Received 2 December 2020 Accepted 30 August 2021 Published Online First 15 November 2021

\begin{abstract}
An understanding of the epidemiology of poststroke dementia (PSD) is necessary to inform research, practice and policy. With increasing primary studies, a contemporary review of PSD could allow for analyses of incidence and prevalence trends. Databases were searched using a prespecified search strategy. Eligible studies described an ischaemic or mixed stroke cohort with prospective clinical assessment for dementia. Pooled prevalence of dementia was calculated using random-effects models at any time after stroke (primary outcome) and at 1 year (range: 6-18 months), stratified for inclusion of prestroke dementia. Meta-regression explored the effect of year of study. Sensitivity analyses removed low-quality or outlier studies. Of 12505 titles assessed, 44 studies were included in the quantitative analyses. At any time point after stroke, the prevalence of PSD was $16.5 \%$ (95\% Cl $10.4 \%$ to $25.1 \%$ ) excluding prestroke dementia and $22.3 \%(95 \% \mathrm{Cl} 18.8 \%$ to $26.2 \%$ ) including prestroke dementia. At 1 year, the prevalence of PSD was $18.4 \%(95 \% \mathrm{Cl} 7.4 \%$ to $38.7 \%$ ) and $20.4 \%(95 \% \mathrm{Cl} 14.2 \%$ to $28.2 \%$ ) with prestroke dementia included. In studies including prestroke dementia there was a negative association between dementia prevalence and year of study (slope coefficient $=-0.05$ (SD: 0.01), $p<0.0001$ ). Estimates were robust to sensitivity analyses. Dementia is common following stroke. At any point following stroke, more than one in five people will have dementia, although a proportion of this dementia predates the stroke. Declining prevalence of prestroke dementia may explain apparent reduction in PSD over time. Risk of dementia following stroke remains substantial and front-loaded, with high prevalence at 1 year post event.
\end{abstract}

\section{INTRODUCTION}

Improving our knowledge of the neuropsychological effects of stroke is of increasing international interest. The 2011 James Lind Alliance, a UK priority setting workshop, identified managing cognitive issues as the most important topic for stroke research. ${ }^{1}$ There is agreement that cognitive problems after stroke are substantial; however, the rates reported vary widely between studies. Previous research has suggested that a history of stroke almost doubles the risk of dementia in the population aged over 65 years. $^{2}$ The comparison and interpretation of these studies are challenging due to differences in study design, that is, duration of follow-up timeframes and the casemix of patients included, for example, combining studies with an intracerebral haemorrhage focus with ischaemic stroke.

A key meta-analysis conducted in 2009 reported that around $10 \%$ of patients had dementia prior to stroke, $10 \%$ developed stroke soon after the first stroke and over 30\% developed dementia after recurrent stroke. ${ }^{3}$ These review data have since been used to inform policy ${ }^{4} 5$ and have informed sample size calculations for studies using poststroke dementia (PSD) as the outcome. ${ }^{6}$ Many studies included in this review are now decades old. In the context of temporal change in dementia prevalence, a new analysis that includes contemporary data seems warranted. The last decade has seen increasing recognition of the importance of PSD with various primary studies on the topic. A contemporary review may offer an estimate of PSD prevalence with greater precision than previous reviews ${ }^{3}$ and would allow for incorporation of risk of bias assessment and framing the certainty of summary results using the Grading of Recommendations, Assessment, Development and Evaluation (GRADE) system.

A consensus approach to PSD diagnosis has been proposed, with recent descriptions from various expert groups describing incident dementia with a temporal relationship to stroke. ${ }^{8} 9$ PSD is part of the spectrum of poststroke cognitive impairment. While PSD has an operationalised definition, variation in definitions and classifications is an issue for other syndromes included in the poststroke cognitive impairment rubric. Limiting a review to PSD allows for a more defined population. Arguably a review that tries to pool data on PSD and milder forms of cognitive impairment risks such heterogeneity that any estimates of prevalence become unhelpful.

An increasingly ageing population coupled with a decline in mortality after stroke ${ }^{10}$ means that PSD may become more prevalent particularly since the risk of stroke and dementia rises exponentially with age. ${ }^{1011}$ Although there are indications of declining incidence of stroke and dementia in developed countries, this may not be the case for developing countries. ${ }^{12}$ Information on PSD prevalence would be useful to provide estimates to design appropriate services to manage the burden of PSD. ${ }^{13}$ As the population prevalence of PSD may show temporal variation, an analysis would allow for exploration of temporal trends.

Therefore, the primary aim of this study was to collate the available evidence to provide a pooled 
prevalence of dementia after ischaemic or mixed stroke. Our secondary aims were to explore subgroups of interest, to assess the effects of study quality and to explore potential heterogeneity in terms of time since stroke, presence of prestroke dementia, recurrent stroke, setting and year of study.

\section{METHODS}

This review was conducted in adherence with the Preferred Reporting Items for Systematic Reviews and Meta-Analyses guidelines ${ }^{14}$ (online supplemental files 2 and 3 ). We used a validated search strategy from an existing review for our primary search. We adapted the search strategy to the focus of our review, limiting to studies of PSD only. We included primary studies published in peer-reviewed journals that included people who had ischaemic stroke, transient ischaemic attack or undifferentiated stroke cohorts and reported quantitative data on the occurrence of PSD at any time point after the stroke event. We accepted any clinical diagnostic assessment, provided it was based on a recognised classification, for example, the Diagnostic and Statistical Manual of Mental Disorders (DSM) ${ }^{15}$ or the International Classification of Diseases (ICD). No restrictions were placed on country, written language or year of publication.

We excluded studies where the primary population of interest was exclusively intracerebral haemorrhage, subarachnoid haemorrhage or traumatic brain injury as all these groups have distinct cognitive recovery profiles. The following types of studies were also excluded: case studies with too few patients to gain reliable conclusions (less than 20 patients); case-control studies and randomised control trials, as they would not give representative population data; and studies that did not use a recognised clinical classification criterion, for example, the use of a single screening tool such as the Mini Mental State Examination or the Montreal Cognitive Assessment without an accompanying clinical diagnostic assessment. Abstracts, letters, editorials and commentaries were also excluded.

\section{Search methods for identification of studies}

Our search syntax used a combination of exploded medical subject headings 'dementia' or 'vascular dementia' or 'multiinfarct dementia' and 'stroke'. ${ }^{3}$ We searched MEDLINE (OVID) and EMBASE (OVID) electronic databases from 2008 (to capture any in press papers that may have been missed in the 2009 search) to July 2019 and conducted forward and backward citation searching of included studies. To identify earlier studies, we assessed the inclusion and exclusion lists of the previous review. We reassessed all studies against our revised inclusion and exclusion criteria. We also hand-searched the following journals for relevant articles published between January 2009 and July 2019: Stroke (American Heart Association), International Journal of Stroke (World Stroke Organization) and European Stroke Journal (European Stroke Organisation). If relevant abstracts were identified but the papers were not available, we contacted the author regarding publication status. Similarly, where relevant data were not available in the published manuscript, we contacted the study authors. As a validation exercise, we cross-checked our selected titles with other reviews that have a stroke cognition focus and no new titles were found. ${ }^{78} 16$

\section{Selection process and data extraction}

All aspects of the selection process were completed by two reviewers (ZLH, TZY). Reviewers were blind to each other's data extraction; data were compared and discrepancies resolved with access to a third arbitrator (TJQ) as required. We extracted data from eligible papers using a prespecified and piloted proforma, based on the Cochrane data extraction tool ${ }^{17}$ and designed to be harmonised with the original study. We collected data on prevalence of dementia (proportion, with corresponding measure of uncertainty), details of the cohort and the methods used to ascertain stroke and dementia status. Where the time point of assessing PSD was not reported, an assumption was derived using the reported dates of cohort inception and the date of paper publication (online supplemental S1,S2).

\section{Data analysis}

The primary outcome for the analysis was prevalence of PSD at any time post stroke, stratified by inclusion or exclusion prestroke dementia. The secondary outcomes were prevalence of PSD at 1 year (allowing for studies with relevant data within a 6-18 months range), stratified by inclusion and/or exclusion of prestroke dementia, and prevalence of prestroke dementia, noting whether prestroke dementia was measured at the time of stroke or only in those patients who survived to follow-up assessment. We calculated point estimates with 95\% CI for all these analyses. Study heterogeneity was expected so we used random-effect models throughout.

\section{Subgroup analysis}

We explored heterogeneity across a series of predefined subgroups of interest: stroke type, which included three levels: first-ever stroke (FES), recurring stroke (RS) or mixed population (cohorts which consisted of both FES and RS populations, yet prevalence data were not reported separately for each population); setting (hospital-based or community-based study); contemporary (published within the previous 10 years) or historical (published more than 10 years ago); and the country's level of income using the WHO classification (high-income or middleincome or low-income country). ${ }^{18}$ These subgroup analyses were conducted for the primary outcome and limited to stroke type for the secondary outcome. Data on PSD were pooled at the following time points: baseline to 3 months, 6 months, 12-18 months, $2-5$ years and $\geq 6$ years. Due to availability of data, the denominators used for the subgroup analyses were those reported for the inception cohort and therefore may vary from the denominators used in the main analysis which were subject to attrition (online supplemental S3-S5).

\section{Sensitivity analysis}

We removed studies considered to be outliers from the metaanalysis to examine the effect on the pooled prevalence. Outliers were identified if the study's CI did not overlap with the CI of the pooled effect. ${ }^{19}$ If the analysis is robust then there should be minimal change in the pooled estimate. ${ }^{20}$ We further performed sensitivity analysis restricting to those studies judged as low or moderate quality.

\section{Meta-regression analysis}

To explore any temporal change in PSD prevalence, we performed a meta-regression of dementia prevalence at any timeframe against the year of study recruitment. Where a cohort was recruited over a longer time period than 1 year, we used the study midpoint. We used Spearman's correlation to test the association between study quality (ordinal sum of risk of bias assessment) and the year of study recruitment. All quantitative analyses were performed using Comprehensive Meta-Analysis V.2.2 (USA) and Statistical Package for the Social Sciences (SPSS) V.26. 
Quality of assessment

We appraised the methodological quality and level of bias using the Newcastle-Ottawa Scale (NOS) for observational studies. ${ }^{21}$ We assessed individual studies including those in the original study using seven relevant items, classified into three categories: the selection of the study groups, the comparability of the groups and the ascertainment of outcome of interest for cohort studies (online supplemental file 3). Points were awarded for each quality item, and the highest quality studies were awarded up to 8 points. No formal cut-offs exist to define low or high risk of bias with the NOS. Therefore, we used cut-offs previously described to form the basis of our scoring system and to pair the score with traffic light coding. ${ }^{22-25}$ Studies with $0-2,3-5$ and 6-8 points represented low, moderate and high quality, respectively. Findings from quality assessment informed a sensitivity analysis limited to studies of high quality.

\section{Publication bias}

Publication bias was assessed by visual inspection of the funnel plots and complemented with statistical testing using Egger's weighted regression and Begg's rank correlation test. $\mathrm{P}<0.05$ was considered to be suggestive of statistically significant publication bias. $^{26}$

\section{Strength of evidence}

An assessment of overall strength of evidence based on the GRADE framework, modified to be suitable for an observational epidemiology question, was performed. ${ }^{27}$ Risk of bias, consistency of results (heterogeneity), directness (applicability of included studies to research question), precision (based on CIs of summary estimate) and publication bias (funnel plot) were all assessed.

\section{RESULTS}

The search identified 12505 articles. After deduplication and screening of titles and abstracts, 128 full-text articles were assessed (figure 1). Data were extracted from 27 studies that meet the eligibility criteria and were included in the review (online supplemental S1-S27). Fifteen studies from the 17 papers included in the previous meta-analysis for PSD prevalence were also included (online supplemental S28-S41, S44). Two studies

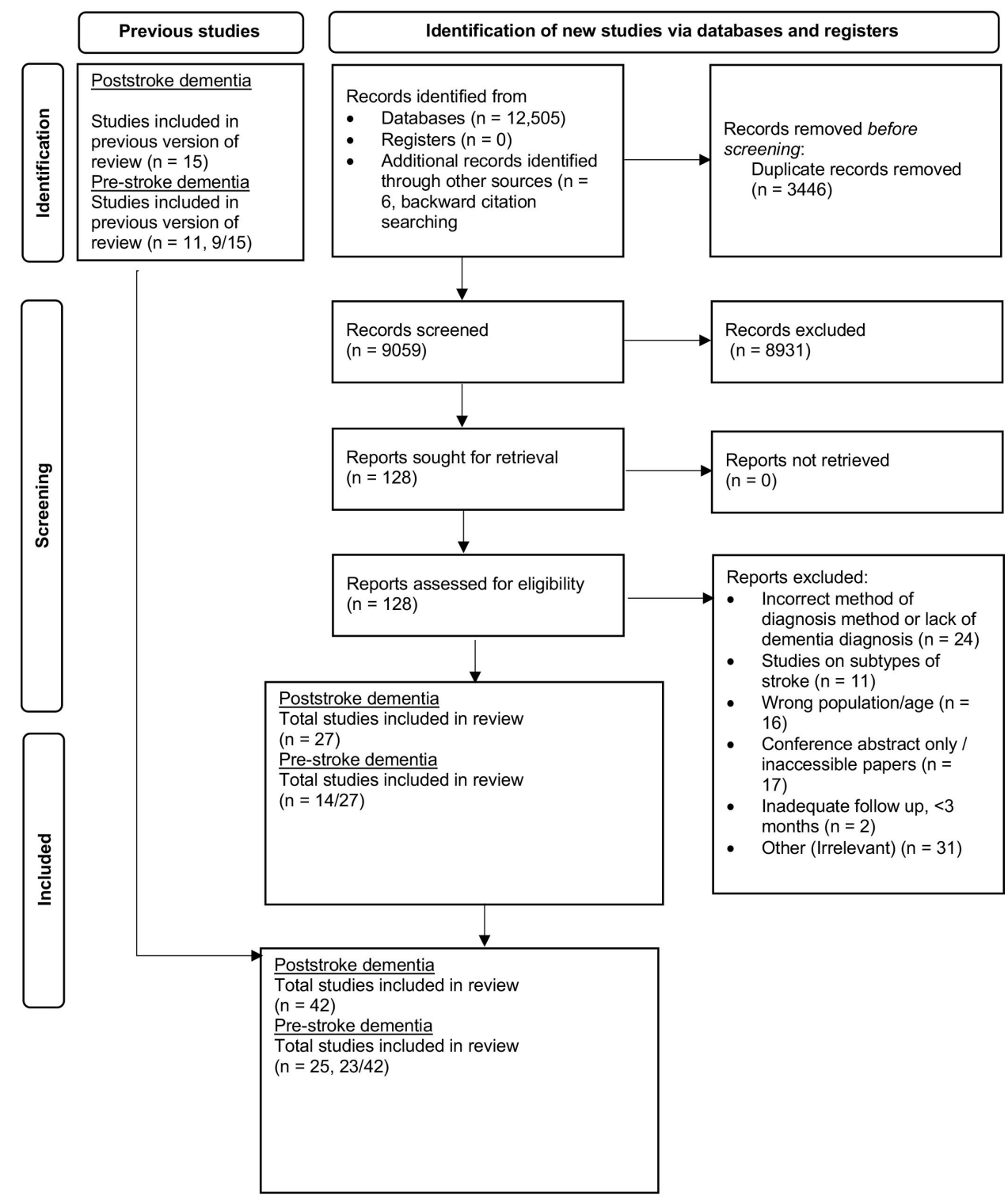

Figure 1 PRISMA search flow. PRISMA, Preferred Reporting Items for Systematic Reviews and Meta-Analyses. 
were excluded due to the method of diagnosis, one study used a screening tool only (online supplemental S43) and the other study, although included in the prestroke analysis, used medical records to identify patients with dementia after stroke (online supplemental S42).

\section{Study characteristics}

The review included 44 studies conducted in the following countries: Africa $(n=5)$, Europe $(n=16)$, Americas $(n=7)$, Asia $(n=15)$ and Australasia $(n=1)$. The sample sizes ranged from 50 to 215118 . The period of follow-up ranged from 3 months to 25 years, with 3 months the modal follow-up time point $(n=18)$. The average age of participants ranged from 56 years to 79.9 years. Most studies used the DSM IV $(n=16)$ (online supplemental S4, S6-S10, S17, S20, S21, S25, S26, S36, S37, S39, S40, $\mathrm{S} 41)$ or the ICD-10 criteria $(\mathrm{n}=8)$ (online supplemental S11, S12, S14, S15, S22, S27, S34, S35). Online supplemental table S1 details the characteristics of the included studies.

\section{PSD prevalence (excluding prestroke dementia): all} timeframes

Data on the prevalence of PSD excluding prestroke dementia regardless of timeframe were available for 24 hospital-based studies (online supplemental S4, S5, S7, S9, S10, S13, S14, S16S18, S20-S23, S25, S26, S28, S29, S31, S33-S35, S37, S44) and for 9 population-based studies (online supplemental S1, S2, S6, S11, S12, S15, S19, S24, S36). The reported prevalence of PSD excluding prestroke dementia in the individual studies ranged from $2.6 \%$ to $39.2 \%$. Based on the 33 studies included in the meta-analysis, the pooled prevalence was $16.5 \%$ (95\% CI $10.4 \%$ to $25.1 \%)$. There were no statistically significant subgroup differences detected (table 1).

Table 1 Prevalence of poststroke dementia: results of the subgroup analysis (all timeframes)

\begin{tabular}{|c|c|c|}
\hline \multirow[t]{3}{*}{ Subgroup (studies, $n$ ) } & \multicolumn{2}{|l|}{ All timeframes } \\
\hline & $\begin{array}{l}\text { Prevalence, \% }(95 \% \mathrm{Cl}), \mathbf{n} \\
\text { studies }\end{array}$ & $\begin{array}{l}\text { Prevalence, } \%(95 \% \mathrm{Cl}) \text {, } \\
\text { n studies }\end{array}$ \\
\hline & $\begin{array}{l}\text { Including prestroke } \\
\text { dementia* }\end{array}$ & $\begin{array}{l}\text { Excluding prestroke } \\
\text { dementia }\end{array}$ \\
\hline \multicolumn{3}{|l|}{ Study setting } \\
\hline Hospital-based & $24.0(20.6$ to 27.8$), n=15$ & $18.2(10.6$ to 29.5$), n=24$ \\
\hline \multirow[t]{2}{*}{ Population-based } & 11.1 (6.3 to 18.7$), n=2$ & 12.4 (4.9 to 28.2$), n=9$ \\
\hline & $P=0.004$ & $P=0.458$ \\
\hline \multicolumn{3}{|l|}{ Stroke type } \\
\hline Recurrent stroke & $41.7(31.9$ to 52.1$), n=4$ & 31.3 (13.9 to 56.4$), \mathrm{n}=8$ \\
\hline First-ever stroke & $18.1(13.3$ to 24.0$), n=5$ & 14.4 (8.3 to 24.0$), n=19$ \\
\hline \multirow[t]{2}{*}{ Mixed population } & $23.8(20.3$ to 27.6$), n=15$ & 19.7 (10.6 to 33.6$), n=14$ \\
\hline & $P=0.000$ & $P=0.270$ \\
\hline \multicolumn{3}{|l|}{ Year of publication } \\
\hline Historical & 27.1 (23.7 to 30.7$), n=11$ & $19.1(8.9$ to 36.3$), n=10$ \\
\hline \multirow[t]{2}{*}{ Contemporary } & $14.4(11.3$ to 18.3$), \mathrm{n}=6$ & 15.1 (8.4 to 25.8$), n=21$ \\
\hline & $P=0.002$ & $P=0.620$ \\
\hline \multicolumn{3}{|l|}{ Level of income } \\
\hline High & $25.4(21.2$ to 30.0$), n=11$ & $12.8(6.9$ to 22.7$), \mathrm{n}=19$ \\
\hline High-middle & 23.5 (15.3 to 35.4$), n=2$ & 19.8 (7.3 to 43.7$), n=7$ \\
\hline Low-middle & $14.9(9.3$ to 22.9$), n=3$ & $25.7(9.9$ to 52.1$), n=7$ \\
\hline \multirow[t]{2}{*}{ Low } & $10.0(4.6$ to 20.5$), n=1$ & No studies \\
\hline & $P=0.014$ & $P=0.414$ \\
\hline
\end{tabular}

* The same study can appear in both inclusion and exclusion of prestroke dementia. Inclusion/exclusion prestroke dementia categories are not mutually exclusive.
Table 2 Prevalence of poststroke dementia: results of the subgroup analysis (1 year)

\begin{tabular}{|c|c|c|}
\hline \multirow[b]{3}{*}{ Subgroup (studies, n) } & \multicolumn{2}{|l|}{ One year } \\
\hline & Prevalence, $\%(95 \% \mathrm{Cl})$ & $\begin{array}{l}\text { Prevalence, } \%(95 \% \mathrm{Cl}) \text {, } \mathrm{n} \\
\text { studies }\end{array}$ \\
\hline & $\begin{array}{l}\text { Including prestroke } \\
\text { dementia }\end{array}$ & $\begin{array}{l}\text { Excluding prestroke } \\
\text { dementia }\end{array}$ \\
\hline \multicolumn{3}{|l|}{ Stroke type* } \\
\hline Recurrent stroke & 33.4 (9.3 to 70.9$), n=7$ & 23.5 (12.7 to 39.1$), n=1$ \\
\hline First-ever stroke & $117.7(6.1$ to 41.9$), n=11$ & $1.0(6.7$ to 17.5$), n=2$ \\
\hline \multirow[t]{2}{*}{ Mixed population } & 28.6 (23.5 to 34.2$), n=5$ & $20.4(4.1$ to 60.4$), n=5$ \\
\hline & $P=0.000$ & $P=0.702$ \\
\hline
\end{tabular}

PSD prevalence (including prestroke dementia): all timeframes

Data on the prevalence of PSD including prestroke dementia regardless of timeframe were available for 14 hospital-based studies (online supplemental S7, S8, S18, S21, S23, S28, S30S32, S34, S37, S38, S40, S44) and for 2 population-based studies (online supplemental S3 and S39). The reported prevalence of PSD including prestroke dementia in the individual studies ranged from $8.4 \%$ to $41.5 \%$. Based on the 17 studies including prestroke dementia, the pooled prevalence was $22.3 \%$ (95\% CI $18.8 \%$ to $26.2 \%)$. Statistically significant subgroup differences were detected for setting $(p=0.004)$, stroke type (first vs recurrent) $(\mathrm{p}<0.001)$, year of publication $(\mathrm{p}=0.002)$ and income $(\mathrm{p}=0.014)$.

PSD prevalence (excluding prestroke dementia): at year 1 Data on the prevalence of PSD excluding prestroke dementia at 1 year following stroke were available for 13 hospital-based studies (online supplemental S4, S5, S10, S13, S14, S17, S20, S22, S25, S31, S33-S35) and for 3 population-based studies (online supplemental S1, S6, S12). The estimated prevalence of PSD in the first year after stroke ranged from $1.1 \%$ to $39.2 \%$. Based on the 16 studies included in the meta-analysis, the pooled prevalence was $18.4 \%$ (95\% CI 7.4 to 38.7 ). No statistically significant subgroup differences were detected (table 2).

PSD prevalence (including prestroke dementia): at year 1

Data on the prevalence of PSD including prestroke dementia 1 year after stroke were available for four hospital-based studies (online supplemental S21, S30, S31 and S34) and for two population-based studies (online supplemental S3, S40). The estimated prevalence of PSD in the first year after stroke ranged from $1.0 \%$ to $31.0 \%$. Based on the six studies included in the meta-analysis, the pooled prevalence was $20.4 \%$ (95\% CI 14.2 to 28.2). A statistically significant subgroup difference was detected for stroke type (first vs recurrent) $(\mathrm{p}<0.001)$ (table 2).

The forest plots for the above meta-analyses are in online supplemental figure $\mathrm{S} 1-\mathrm{S} 8$.

\section{Prestroke dementia}

The pooled prevalence for prestroke dementia was 7.6\% (95\% CI 4.0 to 14.0$)$ ( $n=25$ studies). Data on the prevalence of prestroke dementia were available for 19 hospital-based studies (online supplemental S5, S7-S10, S13, S14, S16, S18, S22, S25, S28, S31, S34, S35, S37, S38, S40, S41) and 6 population-based studies (online supplemental S6, S12, S19, S36, S42, S45). Thirteen of the 19 hospital-based studies obtained rates of prestroke 
dementia at an interview with an informant using the Informant Questionnaire on Cognitive Decline in the Elderly questionnaire. The population-based studies used a variety of methods, such as review of medical records and premorbid assessment of cognition. Eight hospital-based studies assessed all patients who had a stroke on admission to hospital, nine assessed only those patients who survived to follow-up and two studies assessed patients at admission and at follow-up. All population-based studies assessed prestroke dementia before or shortly after stroke.

\section{PSD prevalence by timeframe since stroke}

The studies were classified into the following timeframes: baseline to 3 months, 6 months, $12-18$ months, 2-5 years and greater than 6 years. The pooled prevalence increased from $19.1 \%$ at 3 months to $19.8 \%$ at 6 months and was then lower for each of the later time points (online supplemental figure S9).

\section{Quality assessment}

Thirty-two studies were categorised as high quality and ten studies were categorised as moderate quality. The majority of studies were assessed to have a truly or somewhat representative cohort. Most studies had no age criteria, with a mean age $>60$ years apart from two studies (online supplemental S6 and S16). Studies provided detailed descriptions such as vascular risk factors of the cohort and scored at least 1 point for the comparability section of the NOS. The prevalence estimate appeared lower in high-quality studies than in moderate-quality studies, both for studies that excluded prestroke dementia $(15.3 \%$ $(7.9 \%-27.9 \%)$ vs $20.6 \%(13.7 \%-29.7 \%))$ and studies that included prestroke dementia (21.3\% (17.2\%-26.2\%) vs $25.4 \%$ $(22.6 \%-28.4 \%))$ (table 3).

\section{Sensitivity analysis}

Eight studies were considered to be outliers and were removed from the analysis (online supplemental S1, S2, S4, S10, S12, $\mathrm{S} 17, \mathrm{~S} 26, \mathrm{~S} 44)$, which reduced the prevalence to $15.6 \%(95 \%$ CI $14.0 \%$ to $17.3 \%$ ) for studies excluding prestroke dementia and to $21.4 \%$ (95\% CI $18.2 \%$ to $25.0 \%$ ) for studies including prestroke dementia. Small or large sample sizes (online supplemental S12 and S17) and no specific timeframe for dementia assessment post stroke (online supplemental S1, S2) are likely to be some of the reasons for these outlying results. This reduction of less than $1 \%$ indicates that the analysis was robust when outlier studies were removed. Ten studies considered to be of moderate quality were removed from the analysis (online supplemental S1, S2, S9, S11, S15, S16, S30, S31, S38, S44). This reduced the prevalence by $1 \%$ for studies excluding prestroke dementia and by $0.9 \%$ for studies including prestroke dementia, respectively, indicating that the analysis was robust to the removal of studies of moderate quality (online supplemental table S2).

\section{Publication bias}

The funnel plot analysis suggests publication bias for studies excluding prestroke dementia. For the rank correlation test, Kendall's tau is -0.28 with one-tailed $\mathrm{p}=0.01$. For Egger's test, the intercept is 4.36 , with a $95 \%$ CI from -4.56 to 13.3 , and one-tailed $p=0.16$. The funnel plot analysis suggests publication bias for studies including prestroke dementia. For the rank correlation test, Kendall's tau is -0.29 with one-tailed $\mathrm{p}=0.05$. For Egger's test, the intercept is -3.83 , with a $95 \%$ CI from -7.81 to 0.13 and one-tailed $\mathrm{p}=0.03$ (online supplemental figures S10,S11).
Table 3 Methodological quality assessment of cohort studies using the Newcastle-Ottawa Scale

\begin{tabular}{|c|c|c|c|c|}
\hline Study, year & Selection & Comparability & Outcome & $\begin{array}{l}\text { Total } \\
\text { score } \\
\end{array}$ \\
\hline Akinyemi et al, 2014 S8 & 3 & 1 & 2 & 6 \\
\hline Alteri et al, 2004 s27 & 3 & 2 & 3 & 8 \\
\hline Arauz et al, 2014 S9 & 2 & 1 & 2 & 5 \\
\hline Assayag et al, 2017 S26 & 3 & 1 & 3 & 7 \\
\hline Barba et al, 2000 S28 & 3 & 1 & 2 & 6 \\
\hline Caratozzolo et al, 2016 S10 & 3 & 1 & 3 & 7 \\
\hline Censori et al, 1996 S29 & 3 & 1 & 2 & 6 \\
\hline Clark et al, 2018 S11 & 3 & 0 & 2 & 5 \\
\hline Corraini et al, 2017 S12 & 3 & 2 & 3 & 8 \\
\hline Das et al, 2013 S3 & 3 & 1 & 3 & 7 \\
\hline De Konnig et al, 1998 S30 & 2 & 1 & 2 & 5 \\
\hline De Konnig et al, 2005 S31 & 3 & 0 & 2 & 5 \\
\hline Delgado et al, 2010 S13 & 3 & 1 & 2 & 6 \\
\hline Desmond et al, 2000 S32 & 3 & 2 & 3 & 8 \\
\hline Gorelick et al, 1993 S44 & 2 & 2 & 2 & 6 \\
\hline Gur et al, 1994 S33 & 3 & 1 & 3 & 7 \\
\hline Henon et al, 2001 S34 & 3 & 2 & 3 & 8 \\
\hline Ihle-Hansen et al, 2010 S14 & 3 & 1 & 3 & 7 \\
\hline Inzitari et al, 1998 S35 & 3 & 1 & 3 & 7 \\
\hline Kase et al, 1998 S36 & 3 & 2 & 3 & 8 \\
\hline Khedr et al, 2000 S16 & 2 & 1 & 2 & 5 \\
\hline Kim et al, 2017 S15 & 3 & 0 & 2 & 5 \\
\hline Klimkowitz et al, 2002 S37 & 3 & 2 & 1 & 6 \\
\hline Kokmen et al, 1996 S42 & 2 & 1 & 2 & 5 \\
\hline $\begin{array}{l}\text { Kumutpongpanich et al, } \\
2017 \text { S5 }\end{array}$ & 3 & 1 & 3 & 7 \\
\hline Mehrabian et al, 2015 S17 & 3 & 1 & 2 & 6 \\
\hline Ojagbemi et al, 2017 S18 & 3 & 1 & 2 & 6 \\
\hline Pendlebury, 2019 S6 & 3 & 1 & 3 & 7 \\
\hline Pohjavaara et al, 1997 S38 & 2 & 1 & 2 & 5 \\
\hline Portegies et al, 2016 S19 & 3 & 1 & 3 & 7 \\
\hline Qu et al, 2015 S1 & 3 & 1 & 2 & 5 \\
\hline Renjen et al, 2015 S20 & 3 & 0 & 3 & 6 \\
\hline Sarfo et al, 2017 S21 & 3 & 2 & 3 & 8 \\
\hline Selim et al, 2009 S22 & 3 & 1 & 3 & 7 \\
\hline Srikanth et al, 2004 S39 & 2 & 2 & 3 & 7 \\
\hline Surawan et al, 2018 S4 & 3 & 1 & 3 & 7 \\
\hline Tang et al, 2004 S40 & 3 & 1 & 2 & 6 \\
\hline Tang et al, 2017 S23 & 2 & 1 & 3 & 6 \\
\hline Tu et al, 2013 S24 & 3 & 1 & 2 & 6 \\
\hline Yang et al, 2015 S25 & 3 & 2 & 3 & 8 \\
\hline Yu et al, 2013 S7 & 3 & 1 & 2 & 6 \\
\hline Zhang et al, 2017 S2 & 3 & 1 & 1 & 5 \\
\hline \multirow[t]{2}{*}{ Zhou et al, 2004 S41 } & 3 & & 2 & 7 \\
\hline & \multicolumn{3}{|c|}{ Cut-off score } & \\
\hline Group & Good & Moderate & Poor & \\
\hline Selection & 3 & 2 & 1 & \\
\hline Comparability & 2 & 1 & 0 & \\
\hline Outcome & 3 & 2 & 1 & \\
\hline Total points & $\geq 6$ & 5 & $\leq 4$ & \\
\hline
\end{tabular}

Strength of evidence (GRADE)

The overall strength of evidence for our estimate of dementia prevalence was graded as low due to a high risk of bias and inconsistency (observational heterogeneous studies), publication 
bias and imprecise overall estimate (wide CIs) (online supplemental table S3).

\section{Meta-regression}

There was no significant relationship between the log event rate for dementia prevalence and year of recruitment of the study for studies excluding prestroke dementia (slope coefficient $(\mathrm{SE})=0.03(0.025), \mathrm{p}=0.18)$. There was a significant relationship between the log event rate for dementia occurrence and year of recruitment of the study for studies including prestroke dementia (slope coefficient $(\mathrm{SE})=-0.05$ (0.01), $\mathrm{p}=0.0000$ ). There was no significant association between study quality and year of study recruitment (coefficient $=-0.095, \mathrm{p}=0.55$ ) (online supplemental figures S12 and S13).

\section{DISCUSSION}

This quantitative synthesis of 44 studies suggests that approximately one in five of all stroke survivors have dementia. Risk appears substantial and front-loaded following stroke, with 1 -year prevalence similar to the estimate for dementia at any time point. For this update, we found new evidence that allowed us to offer greater precision in estimates than in previous reviews. Some of our findings confirm the results from other analyses, for example, the rates of PSD were highest in the hospital-based studies of recurrent stroke. ${ }^{3}$ Other findings are unique to our analyses, for example, the apparent decrease in estimates of allcause PSD over time.

The primary aim of this review was to provide a pooled prevalence of PSD and not to examine any relationships between PSD and demographic factors, vascular risk factors or stroke characteristics such as stroke severity. However, through various subgroup analyses we explored factors that may contribute to the high prevalence of PSD. Inclusion of prestroke dementia consistently increased estimates of PSD and emphasises the importance of considering the prestroke state when assessing stroke survivors. Recurrent stroke substantially increased PSD, a finding in keeping with other reviews. Several mechanisms may explain this, including the cumulative impact of neurological insult, underlying cerebrovascular disease or common risk factors. ${ }^{28-30}$ Other sources of heterogeneity relating to casemix and study setting were explored; however, no single factor explained the differences in estimates for studies that excluded prestroke dementia.

Dementia is a progressive condition, yet in our analysis as the length of time between the stroke event and assessment increased, the prevalence of PSD showed a modest decrease. Attrition due to mortality is one plausible explanation. In addition, immediately after the stroke event, there are dynamic changes in cognition and attempts at early assessment may overestimate dementia. It is recommended that any formal diagnosis of dementia is not made until several months after the index stroke. ${ }^{31}$ These estimates of PSD at fixed time points after stroke provide information that can be used for clinical benchmarking, epidemiology and public health messaging.

Our analysis of temporal change in reports of dementia prevalence suggested no change in incident dementia post stroke, but a decrease in PSD when prestroke dementia was included. The factors underlying this are currently not clear. ${ }^{32}$ One plausible explanation is that raised awareness and improved access to diagnosis ${ }^{33}$ have increased the rates of dementia diagnosed before or at the time of the stroke, with a subsequent decrease in poststroke diagnosis. Decreases in all-cause dementia incidence in industrialised countries may also be relevant. Alternatively, it has been speculated that the incidence of vascular dementia may have reduced, supported by the improvement in vascular care. ${ }^{34}$ Although our meta-regression analyses indirectly support this view, caution needs to be drawn when interpreting the causal implications of these analyses. The age-specific risk of all-cause prevalence in the USA and Europe has declined by about 20\% per decade since late 1990s. ${ }^{3536}$ Other factors will also be important and may become more important in the future, for example, improvements in recognition and diagnosis of dementia and increased survival of patients who had a stroke who are at higher risk of developing dementia. For these reasons, it seems prudent to continue to monitor the incidence and prevalence of PSD. In this regard, we look forward to the results of large observational cohorts that are designed to address the interplay of stroke, dementia and vascular disease. ${ }^{37}$

\section{Strengths and weaknesses of this review}

We followed best practice in evidence synthesis and made use of tools such as risk of bias assessment and GRADE to frame our results. There has been a substantial increase in research around stroke and cognition. To keep the review focused and manageable, we limited it to dementia diagnosis, rather than less well-defined syndromes such as poststroke cognitive impairment. A distinct review investigating the prevalence of cognitive impairment no dementia (CIND) has been previously conducted and revealed that in the first year post stroke one in four people present with CIND. ${ }^{38}$ The authors also highlighted that there was significant variation in how the CIND was operationalised, such as the use of different cut-offs for impairment and the use of functional criteria. PSD rather than other cognitive syndromes seems to have the greatest influence on overall prognosis following stroke. ${ }^{39}$ Age is the most important risk factor for dementia ${ }^{40}$ so stratifying the analysis by age groups may have explained the variation in prevalence estimates between the studies. We did not include studies where the primary population of interest was intracerebral haemorrhage, subarachnoid haemorrhage or traumatic brain injury as all these groups have distinct cognitive recovery profiles and combining the various groups may explain conflicting results in previous research (online supplemental S6 and S8).

\section{Areas of future research}

This review has highlighted some important methodological limitations which could be used to inform recommendations for the conduct of future primary research studies in this area, for example, a more inclusive inclusion criteria to overcome the potential underestimation of the prevalence of dementia after stroke. Differing approaches to diagnosis of the dementia syndrome were evident across the papers included in our review. There is ongoing work to standardise diagnosis, such as the consensus statement produced by the Vascular Impairment of Cognition Classification study group. ${ }^{9}$ A recommendation based on this review is for researchers to adopt a standard process or to describe the steps used to diagnose PSD. This would improve the internal and external validity of future observational studies and raise potential for inclusion in future meta-analyses.

Cognitive impairment is associated with increased mortality and morbidity, which can lead to sizeable loss at follow-up impacting of the measurable risk of dementia after stroke. Therefore, future research studies should employ alternative strategies to follow-up and the use of face-to-face assessment such as telephone/video assessment, home visits and postal 
surveys. Furthermore, while the focus of this review was dementia following stroke, there is a need for more epidemiological data on the cognitive consequences of stroke that do not meet the criteria for dementia.

\section{Implications for research and practice}

Our estimates of prevalence allow projections for the future burden of PSD to design appropriate health policy, that is, the allocation of healthcare resources. ${ }^{41}$ Our findings highlight the need for greater engagement between stroke and dementia care. We would hope that our data on prevalence of PSD highlight the importance of this condition among policy makers, healthcare professionals and the public. Our estimates can be used for planning research, for example, in planning the sample size of a future interventional trial.

\section{CONCLUSION}

At all points in the stroke journey, in all healthcare settings and in all countries of the world, PSD is one of the most common complications of stroke. Certain factors were associated with higher prevalence, for example, inclusion of people with prestroke dementia and recurrent stroke, but even when these factors were not present the prevalence remained substantial.

\section{Twitter Terence J Quinn @DrTerryQuinn}

Contributors ZLH and TZY extracted data from included papers. Decisions were cross-checked with TJQ at screening for exclusion and data extraction stages. LEC conducted quality assessment and all analyses. LEC wrote the first draft of the paper with contributions from ZLH, TZY, TJQ and JW. All authors read and approved the final manuscript.

Funding JW receives funding from the UK Dementia Research Institute (which is funded by DRI, funded by the UK Medical Research Council, Alzheimer's Society and Alzheimer's Research UK), the Fondation Leducq (16 CVD 05) and the British Heart Foundation (RE/18/5/34216).

\section{Competing interests None declared.}

Patient consent for publication Not required.

Provenance and peer review Not commissioned; externally peer reviewed.

Supplemental material This content has been supplied by the author(s). It has not been vetted by BMJ Publishing Group Limited (BMJ) and may not have been peer-reviewed. Any opinions or recommendations discussed are solely those of the author(s) and are not endorsed by BMJ. BMJ disclaims all liability and responsibility arising from any reliance placed on the content. Where the content includes any translated material, BMJ does not warrant the accuracy and reliability of the translations (including but not limited to local regulations, clinical guidelines, terminology, drug names and drug dosages), and is not responsible for any error and/or omissions arising from translation and adaptation or otherwise.

Open access This is an open access article distributed in accordance with the Creative Commons Attribution Non Commercial (CC BY-NC 4.0) license, which permits others to distribute, remix, adapt, build upon this work non-commercially, and license their derivative works on different terms, provided the original work is properly cited, appropriate credit is given, any changes made indicated, and the use is non-commercial. See: http://creativecommons.org/licenses/by-nc/4.0/.

\section{ORCID iDs}

Louise Craig http://orcid.org/0000-0003-0057-1949

Joanna Wardlaw http://orcid.org/0000-0002-9812-6642

Terence J Quinn http://orcid.org/0000-0003-1401-0181

\section{REFERENCES}

1 Pollock A, St George B, Fenton M, et al. Top 10 research priorities relating to life after stroke-consensus from stroke survivors, caregivers, and health professionals. Int J Stroke 2014;9:313-20.

2 Savva GM, Stephan BCM, Alzheimer's Society Vascular Dementia Systematic Review Group. Epidemiological studies of the effect of stroke on incident dementia: a systematic review. Stroke 2010;41:e41-6.
3 Pendlebury ST, Rothwell PM. Prevalence, incidence, and factors associated with pre-stroke and post-stroke dementia: a systematic review and meta-analysis. Lancet Neurol 2009;8:1006-18.

4 Eskes GA, Lanctôt KL, Herrmann N, et al. Canadian stroke best practice recommendations: mood, cognition and fatigue following stroke practice guidelines. Int J Stroke 2015:10:1130-40.

5 Royal College of Physicians. National clinical guideline for stroke. 5th edn, 2016.

6 Bath PM, Scutt P, Blackburn DJ, et al. Intensive versus guideline blood pressure and lipid lowering in patients with previous stroke: main results from the pilot 'prevention of decline in cognition after stroke trial' (PODCAST) randomised controlled trial. PLOS One 2017;12:e0164608.

7 Sun J-H, Tan L, Yu J-T. Post-stroke cognitive impairment: epidemiology, mechanisms and management. Ann Trans/ Med 2014;2:80.

8 Mijajlović MD, Pavlović A, Brainin M, et al. Post-stroke dementia - a comprehensive review. BMC Med 2017;15:11.

9 Skrobot OA, Black SE, Chen C, et al. Progress toward standardized diagnosis of vascular cognitive impairment: guidelines from the vascular impairment of cognition classification consensus study. Alzheimers Dement 2018;14:280-92.

10 Rothwell PM, Coull AJ, Giles MF. Change in stroke incidence, mortality, case-fatality, severity, and risk factors in Oxfordshire, UK from 1981 to 2004 (Oxford vascular study). Lancet 2004;363:1925-33.

11 Rothwell PM, Coull AJ, Silver LE, et al. Population-based study of event-rate, incidence, case fatality, and mortality for all acute vascular events in all arterial territories (Oxford vascular study). Lancet 2005;366:1773-83.

12 Feigin VL, Krishnamurthi RV, Parmar P, et al. Update on the global burden of ischemic and hemorrhagic stroke in 1990-2013: the GBD 2013 study. Neuroepidemiology 2015:45:161-76.

13 Béjot $Y$, Aboa-Eboulé C, Durier J, et al. Prevalence of early dementia after first-ever stroke: a 24-year population-based study. Stroke 2011;42:607-12.

14 Moher D, Liberati A, Tetzlaff J, et al. Preferred reporting items for systematic reviews and meta-analyses: the PRISMA statement. Open Med 2009;3:e123-30.

15 American Psychiatric Association. Diagnostic and statistical manual of mental disorders: DSM-5, 2013

16 Hénon H, Pasquier F, Leys D. Poststroke dementia. Cerebrovasc Dis 2006;22:61-70.

17 Higgins JPT, Thomas J, Chandler J. Cochrane Handbook for systematic reviews of interventions version 6.0, 2019.

18 The World Bank. Available: https://datatopics.worldbank.org/world-developmentindicators/stories/the-classification-of-countries-by-income.html [Accessed 2 Dec 2020].

19 Viechtbauer W, Cheung MW-L. Outlier and influence diagnostics for meta-analysis. Res Synth Methods 2010;1:112-25.

20 Bown MJ, Sutton AJ. Quality control in systematic reviews and meta-analyses. Eur J Vasc Endovasc Surg 2010;40:669-77.

21 et alWells GA, Shea B, O'Connell D. The Newcastle-Ottawa scale (NOS) for assessing the quality of nonrandomised studies in meta-analyses, 2008. Available: http://www. ohri.ca/programs/clinical

22 Catalá-López F, Hutton B, Page MJ, et al. Risk of mortality among children, adolescents, and adults with autism spectrum disorder or attention deficit hyperactivity disorder and their first-degree relatives: a protocol for a systematic review and meta-analysis of observational studies. Syst Rev 2017;6:189.

23 Tekelab T, Akibu M, Tagesse N, et al. Neonatal mortality in Ethiopia: a protocol for systematic review and meta-analysis. Syst Rev 2019;8:103.

24 Zulkipli MS, Dahlui M, Jamil Nor'ashikin, Na J, et al. The association between obesity and dengue severity among pediatric patients: a systematic review and meta-analysis. PLoS Negl Trop Dis 2018;12:e0006263.

25 McPheeters ML, Kripalani S, Peterson NB, et al. Closing the quality gap: revisiting the state of the science (Vol. 3: quality improvement interventions to address health disparities). Evid Rep Technol Assess 2012:1-475

26 Egger M, Davey Smith G, Schneider M, et al. Bias in meta-analysis detected by a simple, graphical test. BMJ 1997;315:629-34.

27 Guyatt GH, Oxman AD, Vist GE, et al. GRADE: an emerging consensus on rating quality of evidence and strength of recommendations. BMJ 2008;336:924-6.

28 Pantoni L, Basile AM, Pracucci G, et al. Impact of age-related cerebral white matter changes on the transition to disability -- the LADIS study: rationale, design and methodology. Neuroepidemiology 2005;24:51-62.

29 ladecola C, Park L, Capone C. Threats to the mind: aging, amyloid, and hypertension. Stroke 2009:40:\$40-4.

30 Inzitari D, Pracucci G, Poggesi A, et al. Changes in white matter as determinant of global functional decline in older independent outpatients: three year follow-up of LADIS (leukoaraiosis and disability) study cohort. BMJ 2009;339:b2477.

31 Cerasuolo JO, Azarpazhooh MR, Kapral MK, et al. Evidence of concomitantly increasing stroke and dementia prevalence among those 80 years and older in Ontario, Canada, 2003-04 to 2012-13. Can. J. Neurol. Sci. 2019;46:105-7.

32 Pase MP, Satizabal CL, Seshadri S. Role of improved vascular health in the declining incidence of dementia. Stroke 2017;48:2013-20.

33 Robinson L, Tang E, Taylor J-P. Dementia: timely diagnosis and early intervention. BMJ 2015;350:h3029. 
34 Wolters FJ, Ikram MA. Epidemiology of vascular dementia. Arterioscler Thromb Vasc Biol 2019;39:1542-9.

35 Satizabal CL, Beiser AS, Chouraki V, et al. Incidence of dementia over three decades in the Framingham heart study. N Engl J Med Overseas Ed 2016;374:523-32.

36 Schrijvers EMC, Verhaaren BFJ, Koudstaal PJ, et al. Is dementia incidence declining?: Trends in dementia incidence since 1990 in the Rotterdam study. Neurology 2012;78:1456-63.

37 Wardlaw JM, Doubal F, Brown R, et al. Rates, risks and routes to reduce vascular dementia (R4vad), a UK-wide multicentre prospective observational cohort study of cognition after stroke: protocol. Eur Stroke J 2021:6:89-101.
38 Sexton E, McLoughlin A, Williams DJ, et al. Systematic review and meta-analysis of the prevalence of cognitive impairment no dementia in the first year post-stroke. Eur Stroke J 2019;4:160-71.

39 Leys D, Hénon H, Mackowiak-Cordoliani M-A, et al. Poststroke dementia. Lancet Neurol 2005;4:752-9.

40 Van der Flier WM, Scheltens P. Epidemiology and risk factors of dementia. J Neurol, Neurosur Psych 2005;76:v2.

41 Béjot Y, Aboa-Eboulé C, Durier J, et al. Prevalence of early dementia after first-ever stroke: a 24-year population-based study. Stroke 2011;42:6-1. 